\title{
Lusioersily
}

\section{The Stimulus' Orienting Function May Play an Important Role in IRAP Performance: Supportive Evidence from an Eye-Tracking Study of Brands}

Andrade Ramalho Pinto, J., Vianna de Almeida, R., \& Bortoloti, R. (2020). The Stimulus' Orienting Function May Play an Important Role in IRAP Performance: Supportive Evidence from an Eye-Tracking Study of Brands. The Psychological Record, 70(2), 257-266. https://doi.org/10.1007/s40732-020-00378-2

Link to publication record in Ulster University Research Portal

\section{Published in:}

The Psychological Record

Publication Status:

Published (in print/issue): 30/06/2020

DOI:

10.1007/s40732-020-00378-2

\section{Document Version}

Author Accepted version

\section{General rights}

Copyright for the publications made accessible via Ulster University's Research Portal is retained by the author(s) and / or other copyright owners and it is a condition of accessing these publications that users recognise and abide by the legal requirements associated with these rights.

\section{Take down policy}

The Research Portal is Ulster University's institutional repository that provides access to Ulster's research outputs. Every effort has been made to ensure that content in the Research Portal does not infringe any person's rights, or applicable UK laws. If you discover content in the Research Portal that you believe breaches copyright or violates any law, please contact pure-support@ulster.ac.uk. 


\title{
The Stimulus' Orienting Function May Play an Important Role in IRAP Performance: Supportive Evidence from an Eye-Tracking Study of Brands
}

\author{
Joana Andrade Ramalho Pinto ${ }^{1}$ (D) $\cdot$ Rodrigo Vianna de Almeida $^{1}$ (D) $\cdot$ Renato Bortoloti $^{1,2}$ (D) \\ Published online: 6 February 2020 \\ (C) Association for Behavior Analysis International 2020
}

\begin{abstract}
The Implicit Relational Assessment Procedure (IRAP) has been typically used to access behavioral biases to relations that the participants learned throughout their history of social interactions. Recent research has highlighted the role of the stimulus orienting function on IRAP performance. The present study used the IRAP to assess preference biases for smartphone application (app) icons, namely WhatsApp and Messenger, and intended to check if these biases correspond to the orienting function of these stimuli in a visual search paradigm monitored by eye tracking. The IRAP presented relations between the WhatsApp and Messenger icons and happy or angry facial expressions. The visual search task had two steps: in Step 1, matrices of app icons were designed to emulate smartphone screens, and the participant was instructed to find either the WhatsApp or the Messenger icon; in Step 2, happy or angry faces were presented as targets in matrices of pictures of neutral faces that functioned as distractors. Participants were more prompted to respond Yes than No to the relation between the WhatsApp icon and happy facial expressions in IRAP trials, and showed ambivalent bias to the Messenger icon. Regarding the visual search performance, participants' were faster to find the WhatsApp target than to find the Messenger target. In addition, they were faster to find the happy targets than the angry targets. Results from the current study corroborate the hypothesis that the stimulus orienting function may play an important role on IRAP performance and, as a result, are supportive to the Differential Arbitrarily Applicable Relational Responding Effects (DAARRE) model.
\end{abstract}

Keywords IRAP $\cdot$ eye tracking $\cdot$ WhatsApp $\cdot$ Messenger $\cdot$ facial expressions $\cdot$ orienting function $\cdot$ DAARRE model

Research on consumer experience applies implicit measures to estimate the influence of brands on consumers' attitudes, judgments, and preferences (Maison, Greenwald, \& Bruin, 2001, 2004). The most widely used and well-known procedure is the Implicit Association Test (IAT), which has been

Joana Andrade Ramalho Pinto

joana.andraderp@gmail.com

Rodrigo Vianna de Almeida

rvapsi@gmail.com

Renato Bortoloti

renatobortoloti@gmail.com

1 Universidade Federal de Minas Gerais, Belo Horizonte, Brazil

2 Instituto Nacional de Ciência e Tecnologia sobre Comportamento, Cognição e Ensino (INCT-ECCE), São Carlos, Brazil proposed as a measure of associations between concepts recorded in memory (Greenwald, McGhee, \& Schwartz, 1998). The IAT is based on asking participants to perform a sort of tasks that does not involve confirming or rejecting the belief, preference or attitude under study. Instead, participants are asked to categorize relevant stimuli with positive and negative emotional terms in successive blocks of trials. Thus, on a brand preference IAT, for example, participants are sometimes required to press a left key for a brand 1 (e.g., Coke) and positive words (e.g., tasty), and to press a right key for brand 2 (Pepsi) and negative (e.g., unpalatable) words. If response times are faster on this task than on the other, when categorizing brand 1 with negative and brand 2 with positive, the difference in response times is taken to be an index of Coke over Pepsi relative preference (if the response time difference is reversed, a relative preference index of Pepsi over Coke is assumed). The IAT has become an alternative to research based on explicit self-report measures that can often generate 
biases in results and interpretations (Maison et al., 2004). Although it is useful for a wide range of situations, the IAT effect has some limitations, because it does not provide enough information about the degree to which each stimulus is individually preferred (Barnes-Holmes, Barnes-Holmes, Stewart, \& Boles, 2010a; Barnes-Holmes, Murtagh, BarnesHolmes, \& Stewart, 2010b).

A nonrelative measure of the participant's bias that can benefit research on customer experience is provided by the Implicit Relational Assessment Procedure (IRAP). The IRAP has been typically used to investigate immediate or automatic responses to relations that the participants learned throughout their history of social interaction. The IRAP operates on the assumption that history-consistent response patterns will be emitted more readily than historyinconsistent patterns. It is assumed that the faster the response, the stronger is the participant's attitude towards the relation presented to them. It is hypothesized that the IRAP allows researchers to determine the existence and strength of relations between stimuli in flight (Barnes-Holmes, Barnes-Holmes, Hussey, \& Luciano, 2016). To configure IRAP tasks, the researcher ought to set two classes of label stimuli (say, label 1 and label 2) and two classes of target stimuli (target 1 and target 2), as well as two response-option verbal stimuli. The $2 \times 2$ combination of the two label sets of stimuli and the two target sets of stimuli results in four stimuli relations: label 1 with target 1 , label 1 with target 2 , label 2 with target 1 , and label 2 with target 2 . These relations will occur respectively in trial type $1,2,3$, and 4 . These four trial types will be quasirandomly displayed in two types of block, defined as consistent and inconsistent (i.e., blocks of trials that cohere and do not cohere with the participant's learning history). The deemed correct response-option in the consistent block will be the exact opposite of the deemed correct responseoption in the inconsistent block. This way, the IRAP can check for degrees of relatedness between these sets of stimuli and infer the extent to which previously learned relations interfere with newly required ones, thus providing a possible attitude bias measurement tool. The higher the difference of promptness to respond to one type of relation in one type of block (say, the consistent one), the higher the IRAP effect.

Visual attention has also been taken as a measure of preference bias (Gidlöf, Anikin, Lingonblad, \& Wallin, 2017; Huziwara, de Souza, \& Tomanari, 2016; Milosavljevic, Navalpakkam, Koch, \& Rangel, 2012), and an objective of the current study is to contrast its measure to the preference bias provided by the IRAP. An important measure of visual attention is the speed to direct the gaze towards objects of interest in a visual scene. The individuals' ability to orientate quickly can be investigated through a sequence of visual search tasks. The visual search paradigm consists in finding as fast and accurately as possible a discrepant target in a matrix of stimuli among distractors displayed for limited exposure duration (Frischen, Eastwood, \& Smilek, 2008; Krummenacher, 2006; Treisman \& Gelade, 1980; Yiend, 2010). Several combinations of target and distracters are possible; the simplest and more common one involves a single target within a neutral distracter array (Yiend, 2010). The present study employed the IRAP to assess preference biases for smartphone application (app) icons, namely WhatsApp and Messenger, and intended to check if these biases correspond to stimuli localization quickness in a visual search paradigm as measured by eye tracking.

The IRAP was designed to investigate relations between the WhatsApp and Messenger icons and happy or angry facial expressions. Alongside the IRAP, explicit scales were used to yield other information on participants' preferences. The visual search task had two steps, each involving a single target within matrices of distractors. In Step 1, matrices of app icons were designed to emulate smartphone screens, and the participant was instructed to find either the WhatsApp or the Messenger icon. In Step 2, happy or angry faces were presented as targets in matrices of pictures of neutral faces that functioned as distractors. Thus, the orienting function of the icons and the faces could be contrasted to the IRAP results.

Recent research has been highlighting the role of the stimulus orienting function on IRAP performance (BarnesHolmes, Finn, McEnteggart, \& Barnes-Holmes, 2018; Bortoloti, de Almeida, de Almeida, \& de Rose, 2019; Finn, Barnes-Holmes, \& McEnteggart, 2018). It could be inferred from former conceptual analysis of the IRAP that, if an IRAP performance is indicative of particular histories of relational responding, it might be assumed that the four trial types of the IRAP would be likewise sensitive to these histories. However, a growing body of research suggests that this is not the case. Finn et al. (2018) described, for instance, a pattern of relational responding in which one of the IRAP trial types produces an effect that is significantly larger than the other three. The authors referred to this pattern as Single-Trial-Type-DominanceEffect and proposed the Differential Arbitrarily Applicable Relational Responding Effects (DAARRE) model to account for this and other relational responding patterns (Finn et al., 2018). The DAARRE model proposes that three sources of influence can affect the participant's performance in IRAP tasks: 1) the relationship between the two stimuli presented in each IRAP trial (Crel); 2) the orienting functions of each of these two stimuli themselves (Cfunc); and 3) the coherence functions presented in the response-options, or relational coherence indicators (RCIs; Barnes-Holmes et al., 2018). The current study was designed, and the data were gathered, before very recent developments in the conceptual analysis of the IRAP had been published (in terms of the DAARRE model). However, the study of the relationship between eye movements and IRAP performances appear to be directly relevant to this work. In particular, the DAARRE model has highlighted a potentially important role for the orienting functions of 
the individual stimuli presented within an IRAP and thus testing the relationship between eye movements as orienting responses and IRAP performance is particularly timely.

\section{Method}

\section{Participants}

Twenty individuals ( 12 females and 8 males) were recruited through convenience in a Brazilian university. Their ages ranged from 22 to 49 years $(M=33.75, S D=8.8)$. Fifteen (75\%) were Brazilians, and thus Portuguese native speakers, whereas five $(25 \%)$ were Spanish native speakers from other Latin American countries (Argentina, Colombia, and Mexico). All participants had normal or corrected-to-normal vision. They were naïve to the purpose of the study and gave written informed consent.

\section{Materials and Apparatus}

Explicit measures Participants completed three self-report scales on their usage habits and preference for apps. The first was a discrete ranking scale for preferred apps in which the participants placed whichever app they wanted (most preferred apps ranging from positions 3 to 1 and most disliked apps ranging from positions -3 to -1 ). The second was a five-interval Likert scale (ranging from -2 [dislike a lot] to 2 [like a lot]) constructed to identify participants' likeability of specific instant messaging apps, namely WhatsApp and Messenger. The third was SelfAssessment Manikin (SAM), an intercultural pictorial seven-interval scale based on three affective dimensions: valence, arousal, and dominance (Bradley \& Lang, 1994). In this study, two affective dimensions were considered: valence (from -3 [negative] to 3 [positive]) and arousal (from -3 [passive] to 3 [active]). The SAM scales were applied not only to both apps but also to one instance of the happy face pictures and one instance of the angry face pictures, to check if participants reported emotional relation to these images, because they were used in the IRAP trial types (described below).

The Implicit Relational Assessment Procedure (IRAP) On each IRAP trial, a sample, a target, and two response options were displayed on a computer screen. The sample-either the WhatsApp or the Messenger icon-was presented on the top of the screen; a single picture target - a happy or an angry face-appeared at the center; and the response options-Sim (for Yes) and Não (for No) - were displayed at the two bottom corners of the screen (Sim on the left and Não on the right). In this arrangement, the following four trial types were presented in each IRAP block: WhatsApp-
Happy face (trial type 1), WhatsApp-Angry face (trial type 2), Messenger-Happy face (trial type 3) and MessengerAngry face (trial type 4). Four pictures of happy faces and four pictures of angry faces were chosen from the Pictures of Facial Affect database (Ekman \& Friesen, 1976). They alternated as targets throughout the IRAP trials.

The IRAP trials were divided into pro-WhatsApp/antiMessenger and pro-Messenger/anti-WhatsApp blocks. The pro-WhatsApp/anti-Messenger verbal rule presented before starting the consistent blocks was the Portuguese version of "Classify WhatsApp in a POSITIVE way (happy faces) and Messenger in a NEGATIVE way (angry faces)." The proMessenger/anti-WhatsApp verbal rule presented before starting the inconsistent blocks was "Classify WhatsApp in a NEGATIVE way (angry faces) and Messenger in a POSITIVE way (happy faces)."

Visual search measures An eye-tracking device was used to record target localization time. The Eye Tribe tracker (www.github.com/EyeTribe) generated raw gaze point location data. The device is a noninvasive binocular recorder and works with infrared (NIR) detection. It operates $60 \mathrm{~Hz}$ sampling rates with a $0.5^{\circ}-1^{\circ}$ average accuracy. The Eye Tribe was connected and positioned below the screen of a laptop with a 15 -inch screen and $1920 \times$ 1080 resolution, at $65 \mathrm{~cm}$ of distance between the participant and the display. Stimulus presentation and data collection were controlled by OGAMA 5.0 open-source software (www.ogama.net).

\section{Procedure}

The participants started by filling a ranking scale with their three preferred and three most disliked apps. Then, they used a Likert scale to rate the likeability of WhatsApp and Messenger. Finally, the SAM affective scales (valence and arousal) were applied to assess the affective reaction to happy and angry faces, which were used as targets in the IRAP procedure, and WhatsApp and Messenger icons, which were used as labels. After completing the explicit measures, participants were instructed to work on a computer programmed with IRAP software.

The IRAP trials were divided into pro-WhatsApp/antiMessenger and pro-Messenger/anti-WhatsApp blocks of trials. In all trial, the app label, the face target and the Yes and No response options (RCIs) remained visible until the participant pressed one of the response keys. The task consisted of choosing one of these options by pressing either the $d$ or the $k \mathrm{key}$, corresponding to Yes or No, respectively. The choice of the option considered correct removed all stimuli from the screen and, after $400 \mathrm{~ms}$, the next trial was presented. The choice of the option considered incorrect produced a red $\mathrm{X}$ in the middle 
of the screen (immediately below the target picture). The next trial was presented only after the participant pressed the correct key.

All participants were presented with blocks of 24 trials - at least two practice blocks and six test blocks. The practice blocks were repeated until at least $80 \%$ correct choices had been made consecutively in one consistent and one inconsistent block. Within each block, the target stimulus could be either a happy face or an angry face, in a randomized sequence, with the restriction that the target was happy in $50 \%$ of the trials and nonhappy in the other $50 \%$. The happy face could be one of the four pictures in this category, in a randomized order, so that each of the specific happy faces appeared four times. The same applied to the angry faces. In the consistent blocks, trials that presented the WhatsApp icon as sample and a happy face as target, and trials that presented the Messenger icon as sample and an angry face as target, both demanded the choice of the option Yes, whereas No was the correct choice in trials that presented WhatsApp as sample and an angry face as target, and in trials that displayed Messenger as sample and a happy face as target. Incorrect choices caused a red $\mathrm{X}$ to be presented below the face, and the participant had to make the correct selection in order to advance to the next trial. In the inconsistent blocks, the opposite responses were required. Fig. 1 illustrates the four different trial types in this part of the experiment.

After completion of all practice blocks and the six test blocks, a written message indicated the end of this experiment phase. Then the participant started the visual search tasks, in the same computer.

Participants performed two visual search tasks synchronized with the eye-tracking system. Gaze starting point was in the middle of the screen and was at the same spot throughout the entire experiment. Each participant performed a nine-point calibration before beginning the experiment. The OGAMA software was launched and the data recording started immediately after the calibration process.

The eye-tracking tasks stimuli consisted of $6 \times 4$ matrices with equally spaced elements. In the Visual Search Task 1, participants should find either the WhatsApp or the Messenger icon among 23 distracting app icons in an arrangement that simulated a smartphone screen. In the Visual Search Task 2, participants should find either a happy or an angry face among 23 distracting neutral faces. Twenty-four matrices were designed for each task. All matrices had the target stimuli in one of the 24 possible stimulus spots. There were 48 search trials (target in each matrix position, twice) and a display maximum duration of 5,000 ms exposure for each search trial.

In the Visual Search Task 1, participants were instructed to "Find and click on the WhatsApp or Messenger app as fast as possible." This experiment consisted of a visual search on a 6 $\times 4$ matrix of app icons, through 48 trials. The distractors in this experiment were different popular app icons (e.g. Facebook, Instagram, Linkedin, among others) simulating a main navigational interface of an iPhone 7 smartphone. The distractor apps were maintained in fixed locations over the 48 trials. Each target app icon was presented in each position of the $6 \times 4$ matrix, yielding 24 possibilities.

In the Visual Search Task 2, participants were instructed to "Find and click on the HAPPY or ANGRY face as fast as possible." Examples of matrices of stimuli used in tasks 1 and 2 are presented in Fig. 2.

Each trial began with a red dot at the center of a white background, for $2,000 \mathrm{~ms}$, followed by a matrix. Matrices were removed after a click on the target or $5,000 \mathrm{~ms}$, whichever came first. Reaction time (RT) in trials in which participants failed to click on the target in time were registered as $5,000 \mathrm{~ms}$. Intertrial interval was $2,000 \mathrm{~ms}$.

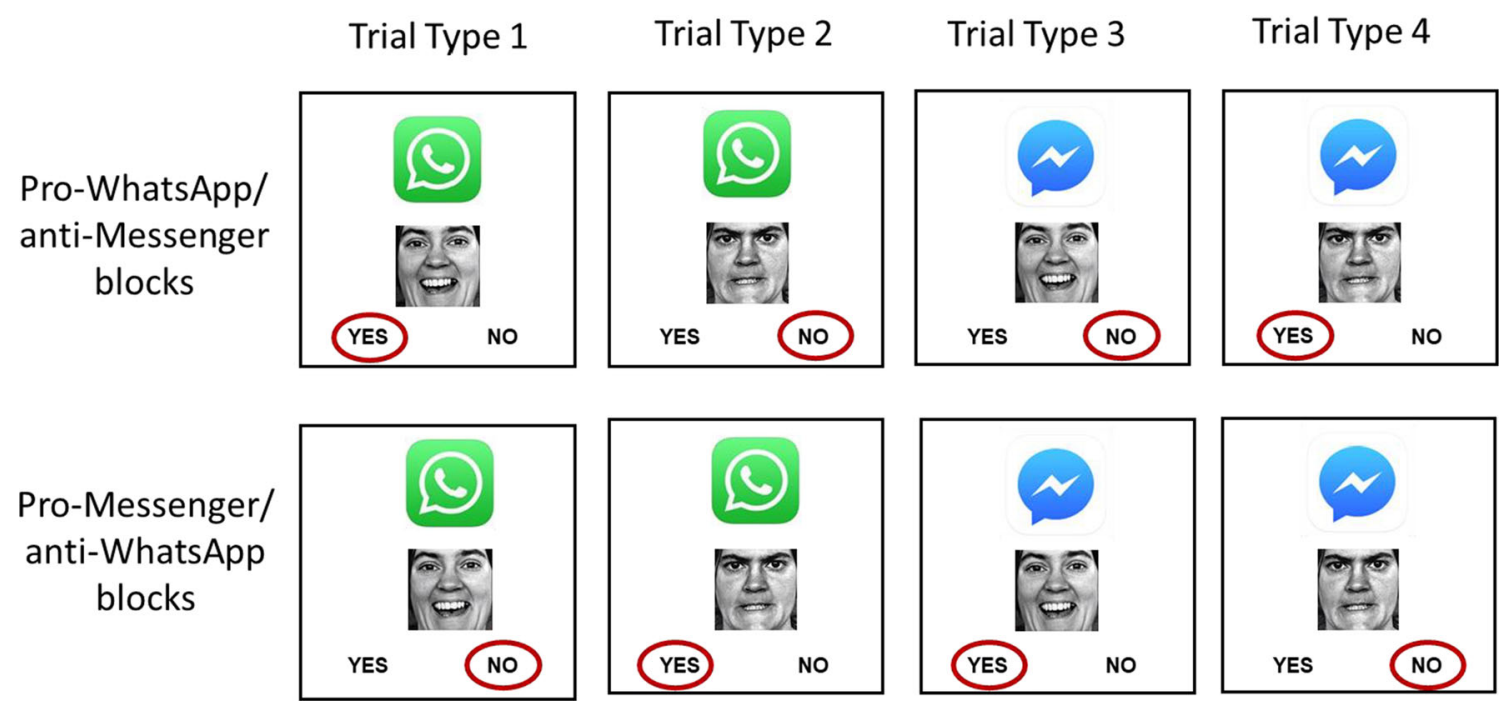

Fig. 1 Schematic illustration of the four trial types presented during the IRAP blocks. 


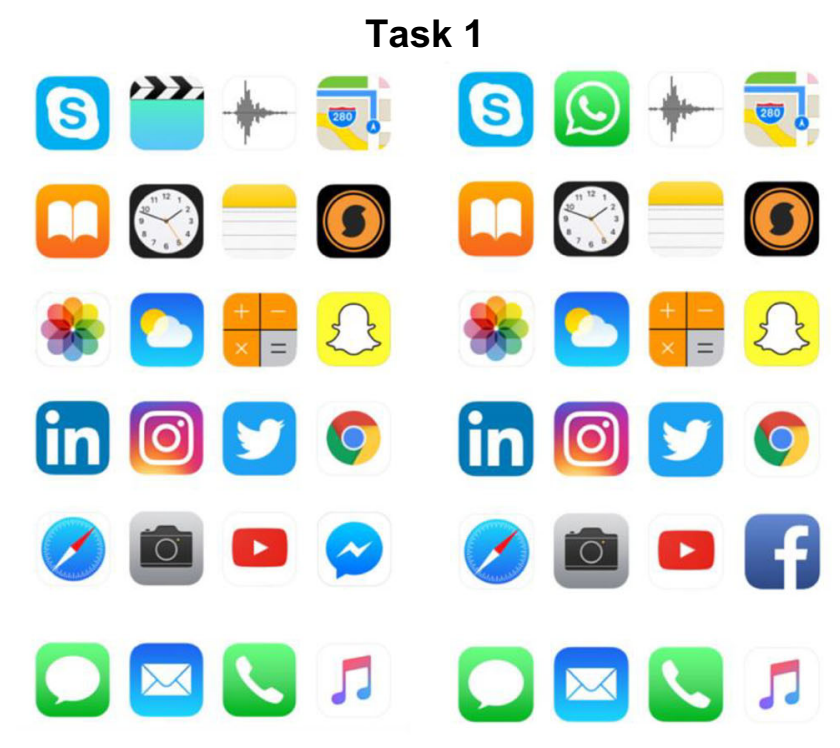

Task 2
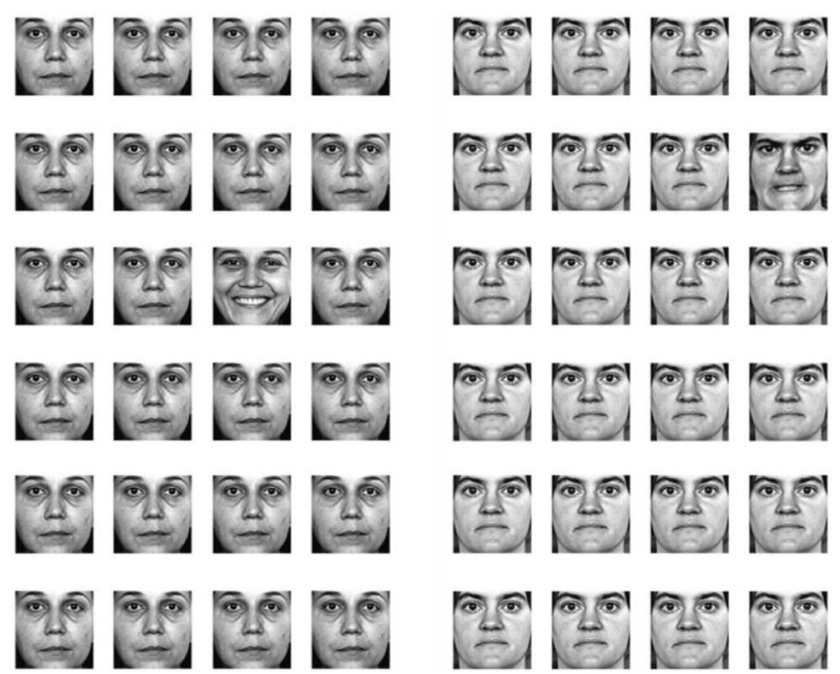

Fig. 2 Examples of visual matrices used in the experiment. Task 1: Distracters were different popular app icons, and targets were either the Messenger icon (on the left) or the WhatsApp icon (on the right). Task 2: Distracters were neutral faces, and targets were either a happy (on the left) or an angry (on the right) face; all faces in a matrix were portrayed by the same female person.

\section{Data Analyses}

Descriptive statistics were used to analyze the distribution of the explicit scales, such as means and standard deviations.

For analyzing the IRAP performances, the IRAP software converts response times into D-IRAP scores (Barnes-Holmes et al., 2006), whose algorithm is based on the IAT's $D$ measure algorithm. The steps to obtain the D-IRAP scores are: (1) only response time data from test blocks (not from practice blocks) are considered; (2) response time latencies above 10,000 ms are eliminated; (3) all data from a participant are removed if they produced time under $300 \mathrm{~ms}$ in over $10 \%$ of test-block trials; (4) twelve standard deviations for each of the four trial types are calculated: four to response times in test blocks 1 and 2 , four to response times in test blocks 3 and 4 , and four to response times in test blocks 5 and 6 , uniting thus one consistent and one inconsistent test blocks in each pair; (5) twentyfour mean response times are calculated to each of the four trial types in each test block; (6) difference scores are calculated to each of the four trial types, for each test blocks pair (consistent and inconsistent), subtracting the consistent block mean response time from the inconsistent block mean time; (7) each difference score is divided by its standard deviation obtained in step "d," yielding 12 D-IRAP scores: one for each trial type for each test-block pair; (8) four general D-IRAP scores relative to the four trial types are obtained from the mean among the three test-block pairs regarding each of the four trial types.

It is thus important to understand that the D-IRAP score calculation relies strongly on subtracting the consistent blocks' mean response time from the inconsistent blocks' mean response time. This "direction" of the subtraction is responsible for the fact that positive D-IRAP scores reflect a proconsistent relations behavioral bias, and a negative D-IRAP score reflects a proinconsistent relations bias. The relativization (division by the pooled standard deviation) is important because the reaction times could otherwise be explained by confounding individual differences, such as intelligence (O'Toole \& Barnes-Holmes, 2009).

The variables from the eye-tracking tasks were the latency time to find the target app (within up to the 5,000 ms limit) and the number of gaze fixations. Data were extracted from the OGAMA 5.0 open-source software, which enables the recording and processing of eye movements and reaction time, i.e., time to click on the target (RT). A key step in processing eyetracking data is the identification of eye-tracking metrics, such as fixations and saccades. The spatial threshold is based on a circle and parameters used for eye movements were $100 \mathrm{~ms}$ as minimal fixation duration and 40 pixels ( $1.1^{\circ}$ visual angle).

Data were evaluated through statistics and graphics analyses. Statistics were calculated for the following variables: fixations, saccades, views and mouse movements. Visual graphics could be constructed from the main modules in OGAMA, such as Attention Map, Area of Interest (AOI), Fixations, and Scanpaths. The Attention Map Module (heat map) is based on the number of fixations. Fixation parameters are presented by number, length, recorded $\mathrm{X}$ and $\mathrm{Y}$ coordinates, and count threshold over $100 \mathrm{~ms}$; Scanpath presents the eye movement route; and Areas of Interest (AOI) is a specific area defined on the image to be observed.

Raw data obtained in this study and some analyses can be found at https://osf.io/qnvbh/ and through the DOI 10.17605/ OSF.IO/QNVBH. 


\section{Results}

\section{Explicit Preference Measures}

According to participants' reports, the frequency of WhatsApp usage was higher than the frequency of Messenger usage: all participants reported daily use of WhatsApp and $75 \%$ of them reported using Messenger eventually (only $45 \%$ reported daily use of Messenger). In addition, the participants showed biases that are more positive for WhatsApp relative to Messenger in the explicit measures used in the current study. Descriptive statistics (alongside mean difference hypothesis tests) of each explicit preference measure are presented in Table 1.

WhatsApp's valence was very positive or positive for $50 \%$ of participants (they rated 2 or 3 ), slightly positive for $25 \%$ (rated 1), and indifferent for 20\% (rated 0). Messenger's valence was very positive or positive for $15 \%$, slightly positive for $20 \%$, and indifferent for $50 \%$. Arousal for WhatsApp was $50 \%$ positive (rated 1,2 , or 3 ) and $50 \%$ negative or indifferent (rated $0,-1,-2$, or -3 ). Arousal for Messenger was $25 \%$ positive (above 0 ) and $75 \%$ indifferent or negative (equal to, or smaller than 0 ).

Valence for happy faces (not presented in Table 1) was $65 \%$ positive and none negative $(M=2.85, S D=.98)$, whereas valence for angry faces was $50 \%$ negative and none positive $(M=-.75, S D=1.06)$. Therefore, participants rated happy faces more positively. Arousal for happy faces had $M=1.1$ and $S D=2.07$, whereas arousal for angry faces had $M=1.4$ and $S D=1.82$, which yields inconclusive interpretations.

\section{The Implicit Relational Assessment Procedure (IRAP)}

Seventeen participants achieved the practice criteria after the training blocks. Individual response latency data were transformed to D-IRAP scores using the D-IRAP algorithm. Positive D-IRAP scores indicate that the participants responded faster in the pro-WhatsApp/anti-Messenger blocks, whereas negative D-IRAP scores indicate faster responding in the pro-Messenger/Anti-WhatsApp blocks.

Table 1 Explicit Preferences measures.

\begin{tabular}{llll}
\hline & WhatsApp & Messenger & p-value \\
\hline Preferences measures & M (SD) & M (SD) & \\
Ranking scale (-3 to 3) & $2.27(1.23)$ & $-1.0(1.07)$ & 0.0001 \\
Likert scale (-2 to 2) & $1.50(0.83)$ & $0.23(1.03)$ & 0.0006 \\
SAM Valence & $1.45(1.23)$ & $0.20(1.44)$ & 0.005 \\
SAM Arousal & $-0.05(2.01)$ & $-0.65(1.60)$ & 0.303 \\
\hline
\end{tabular}

Note: Mean (M), standard deviation (SD) and difference between WhatsApp and Messenger explicit preferences measures
Figure 3 presents participants' mean D-IRAP scores for each of the four trial types.

The mean D-IRAP score for the WhatsApp-Happy trial type had the highest scores and was significantly different from zero $[t(16)=8.19, p<0.0001]$, which indicates a strong behavioral bias when relating the WhatsApp icon with a happy facial expression. The WhatsApp-Angry trial type presented a positive D-IRAP score, which was roughly significant $[t(16)=1.77, p=0.09]$. This result indicates that participants were faster to deny the relation between WhatsApp and angry faces. The Messenger-Happy trial type had a negative mean D-IRAP score, indicating that participants responded faster to the pro-Messenger blocks (i.e., they were faster to relate the Messenger icon to happy facial expressions with a Yes cue). However, this value is not significantly different from zero $[t(16)=-1.44, p=0.1694]$. The Messenger-Angry trial type had a D score indicating that the participants spent similar time in the consistent and inconsistent blocks $[t(16)=0.42, p=$ 0.6749].

A repeated measures ANOVA showed a significant effect for the difference between the mean D-scores that participants produced for the IRAP trial types (F [3 16] $=10.94, p<$ 0.0001). To extend the interpretation of this result, a TukeyKramer multiple-comparison test was conducted and the results of this post-hoc test are presented in Table 2. Significant differences were observed between the mean D-IRAP scores only from: WhatsApp-Happy (trial type 1) versus WhatsAppAngry (trial type 2); WhatsApp-Happy (trial type 1) versus Messenger-Happy (trial type 3); WhatsApp-Happy (trial type 1) versus Messenger-Angry (trial type 4).

\section{Visual Target Localization Measures}

The variables from the visual search task were the time to find the target and the number of gaze fixations. Data from all the 20 participants were analyzed. Figure 4 presents the mean reaction time for WhatsApp and Messenger targets and the mean number of gaze fixations before finding WhatsApp and Messenger targets.

The mean reaction time to WhatsApp targets was significantly smaller than reaction time to Messenger targets $[t(19)=$ $2.534, p=0.02]$. A similar difference pattern was found for the number of gaze fixations the participants performed before finding the targets among the distractors. The mean number of fixations before finding the WhatsApp targets was smaller than the number of gaze fixations before finding the Messenger targets, difference that was roughly significant $[t(19)=2.15, p=0.044]$.

Figure 5 presents the mean reaction time for happy and angry targets and the mean number of gaze fixations before finding happy and angry targets.

The mean reaction time to happy targets was significantly smaller than reaction time to angry targets $[t(19)=12.20, p<$ 
Fig. 3 Mean D-IRAP scores for each trial type extracted from the performances of the 17 participants who achieved both the IRAP criteria.

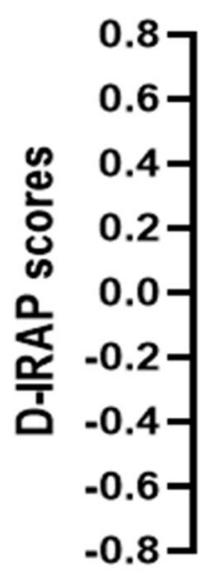

0.0001]. A similar difference pattern was found for the number of gaze fixations the participants performed before finding the targets among the distractors. The mean number of fixations before finding the happy targets was significantly smaller than the number of gaze fixations before finding the angry targets $[t(19)=6.321, p<0.0001]$.

\section{Discussion}

An important objective of the current study was to contrast results of three measures of biases towards two widely known application icons. The explicit measures clearly show a higher pro-WhatsApp bias. Agreeing to the explicit measures, the IRAP results proved participants to be much more prompted to respond Yes than No to the relation between the WhatsApp icon and happy facial expressions. Regarding the Messenger icon, participants did not show a strong attitude pro or anti this app in the explicit scales, which is likewise reflected in the Messenger-Happy and Messenger-Angry trial types. Regarding the visual search performance (which can be

Table 2 Results from the Tukey-Kramer Multiple Comparisons Test considering the four IRAP trial types. If the value of $\mathrm{q}$ is greater than 3.65 , then the $\mathrm{P}$ value is less than 0.05 .

\begin{tabular}{lllr}
\hline Comparison & $\begin{array}{l}\text { Mean } \\
\text { difference }\end{array}$ & $\mathrm{q}$ & $\mathrm{p}$ value \\
\hline Type 1 vs. Type 2 & 0.3441 & 3.811 & 0.0434 \\
Type 1 vs. Type 3 & 0.6875 & 7.614 & $<0.0001$ \\
Type 1 vs. Type 4 & 0.5484 & 6.073 & 0.0003 \\
Type 2 vs. Type 3 & 0.3434 & 2.866 & 0.2195 \\
Type 2 vs. Type 4 & 0.2043 & 2.262 & 0.3862 \\
Type 3 vs. Type 4 & -0.1391 & 1.540 & 0.6974 \\
\hline
\end{tabular}

taken as a measure of preference bias; Gidlöf et al., 2017; Huziwara et al., 2016; Milosavljevic et al., 2012), participants' reaction time (RT) to the WhatsApp target was smaller than their RT to the Messenger target, and the mean number of gaze fixations before finding the WhatsApp target was also smaller than the number of gaze fixations before finding the Messenger target. In addition, the RT to the happy targets was smaller than the RT to the angry targets, and consistently the mean number of gaze fixations before finding the happy targets was smaller than the number of gaze fixations before finding the angry targets. As far as we know, this is the first study to present a set of data contrasting performances in IRAP and visual search tasks. In the following paragraphs, we examine these results in detail and discuss their implications.

Inspecting the IRAP data we can observe an effect that has been referred to as Single-Trial-Type-Dominance-Effect (STTDE; Finn et al., 2018). In particular, the D-score for the trial type 1 (WhatsApp icon and happy faces) was significantly larger than the D-scores for the other three trial types. Finn et al. (2018) reported the STTDE in an IRAP in which labels were either the words color or shape, and targets were words denoting colors or shapes. Significantly larger D-IRAP scores were found for the trial type that presented the label color and color words. This was attributed to stronger orienting functions of color words, due to their higher frequency of occurrence in the language compared to shape words. Consistent with this explanation, the STTDE did not occur in an IRAP in which labels were words with approximately equal frequency in the language, spoon and fork, and the targets were pictures of spoons and forks. This was explained by equally strong orienting functions of these stimuli. The expression orienting function was used to suggest that in general colors would be more salient or attention-grabbing than 
Fig. 4 Mean reaction time and mean number of gaze fixations to find either the Messenger or the WhatsApp targets among other icons presented as distractors.

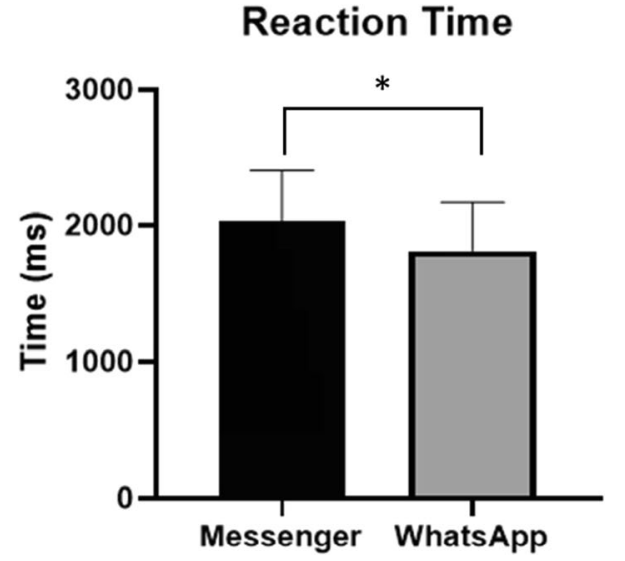

Gaze Fixation

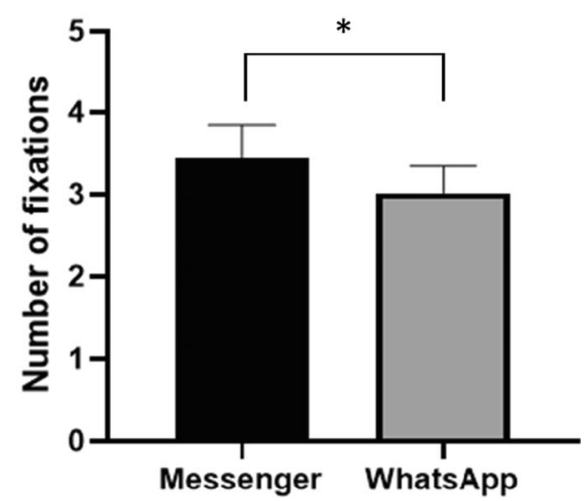

forms, and that such a difference would be absent among spoons and forks. This is a plausible hypothesis, but it was articulated based on a post-hoc analysis of IRAP performances, without any direct measure of the orientation function of these stimuli as we did in the current study by means of visual search tasks. Thus, the current findings appear to be relevant to a recent conceptual analysis of the IRAP that is briefly described below.

Finn et al. (2018) proposed the Differential Arbitrarily Applicable Relational Responding Effects (DAARRE) model to account for the STTDE and other relational responding patterns. The DAARRE model proposes that three sources of influence can affect the participant's performance in IRAP tasks: the relationship between the two stimuli presented in each IRAP trial (Crel), the orienting functions of each of these two stimuli themselves (Cfunc), and the coherence functions presented in the response-options, or relational coherence indicators (RCIs; Barnes-Holmes et al., 2018). The DAARRE model highlighted an important role for the orienting functions of the individual stimuli presented within an IRAP and thus it seems appropriate contrasting visual search as orienting responses and IRAP performance.

Figure 6 presents a schematic representation of the DAARRE model as it applies to the app icons and facial expressions IRAP. Consistent to the current visual search data, we assume that the WhatsApp icon and the happy faces possess stronger orienting function than the Messenger icon and the angry faces respectively. In Fig. 6, the Cfuncs for the WhatsApp icon and the happy faces are labeled as positive and the Cfuncs for the Messenger icon and the angry faces are labeled as negative. The negative labeling for Messenger and angry faces should not be taking to indicate a negative orienting function, but only an orienting function that is weaker than that of WhatsApp and the happy faces. The labeling of the relations between the icon (label) and face (target) stimuli indicates the extent to which they cohere or do not cohere based on the participants' responses to the explicit measures (and also inferred from their visual search performance). Thus, a WhatsApp-happy face relation is labeled with a plus sign (i.e., coherence), a WhatsApp-angry face relation is labeled with a minus sign (i.e., incoherence), whereas both the Messenger-happy face and the Messenger-angry faces relations are labeled with plus and minus signs (i.e., undetermined coherence level or ambivalence). Finally, the two response options (RCIs) - the Portuguese words equivalent to Yes (Sim) and No (Não) — are each labeled with a plus or minus sign. Because Yes serves frequently as a confirmatory, favorable, or positive response in natural language (i.e., indicates coherence), it is labeled with a plus sign; because No is often used to indicate incoherence, it is labeled with a minus sign.
Fig. 5 Mean reaction time and mean number of gaze fixations to find either happy or angry targets among neutral distractors.
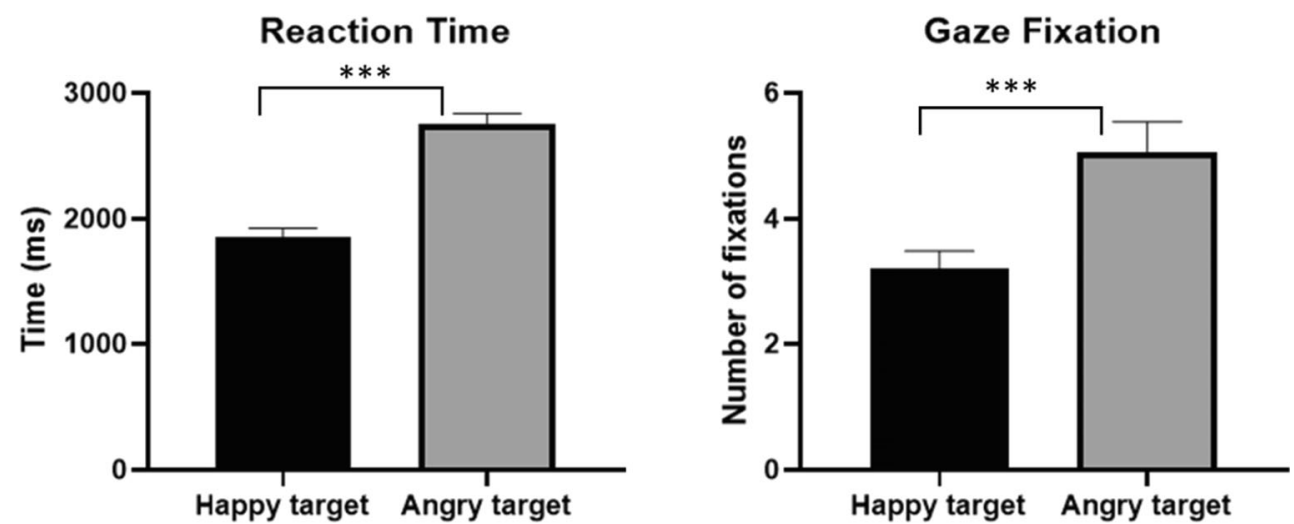


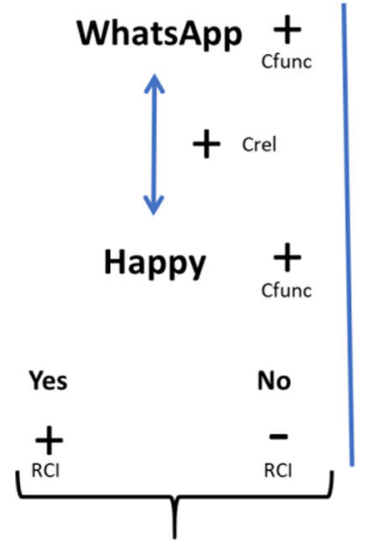

Max coherence

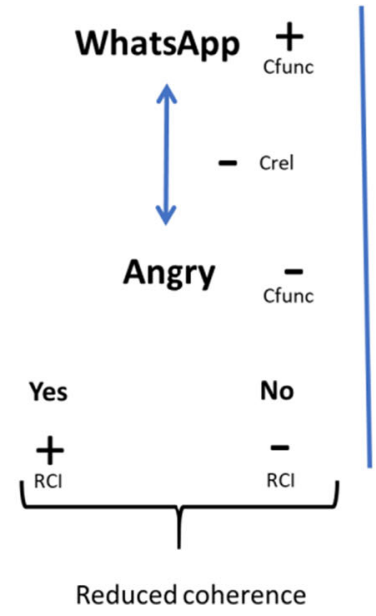

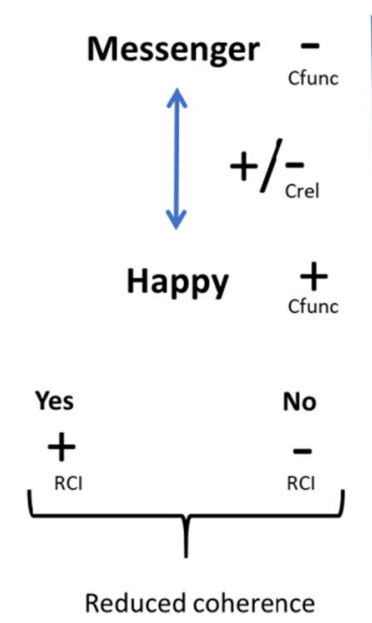

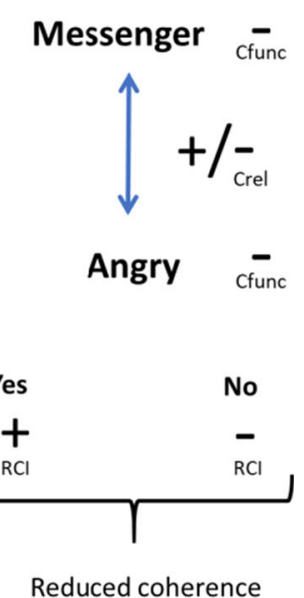

Fig. 6 The DAARRE model as it applies to the app icons and facial expressions IRAP.

As presented in Fig. 6, each trial type differs in terms of plus and minus properties of Cfuncs and Crels. Note that these labels are based on current behavioral measures, and they should not be seen as inherent to the stimuli themselves. They are useful in explaining the STTDE of the trial type 1 and the other IRAP effects as well. Regarding the trial type 1, the Cfunc and Crel properties are all labeled with plus signs, and the RCIwhich is presumably consistent to the participants' history (inferred from both the explicit and visual search measures) - is also labeled with a plus sign. According to the DAARRE model, this trial type may be considered to be of the maximum coherence during history-consistent blocks; during the history-inconsistent blocks, however, there is no coherence between the RCI and the properties of the Cfuncs and Crels, which are all labeled with plus signs in Fig. 6. The high contrast regarding the level of coherence across history-consistent and historyinconsistent blocks may have been determinant to the large IRAP effect in the trial type 1, an effect that is consistent to the DAARRE model.

The IRAP effects presented in Fig. 3 are consistent with the explicit and visual search measures. Summing up the behavioral patterns that participants showed for each trial type, they confirmed much more readily than denied a relation between the WhatsApp icon and happy faces (trial type 1) as outlined above, and tended to deny more readily to confirm a relation between WhatsApp and angry faces (trial type 2). They also failed, at a statistically significant level, to deny more quickly to confirm, or to confirm more quickly to deny, relations between the Messenger icon and both the happy (trial type 3) and angry faces (trial type4). All of these IRAP effects are consistent to the DAARRE model, as it is represented in Fig. 6.

A possible limitation of the scope of data reported in this article regards to the number of participants, which in the present study is relatively small. In addition, a potential source of confounding is the fact that not all participants were native Portuguese speakers. Even living for a long time in Brazil, and being fluent in Portuguese, it should be considered that the presumed Cfunc properties of the RCIs Yes and No might somehow be affected by experience with the language (i.e., possibly Sim and Não do not have the same strength as $S i$ and No for a native Spanish speaker). Such issues should be explored in further investigation, which should also consider testing the potential influence of labels with similar orienting effects, as determined in visual search tasks, on the IRAP effects in different trial types.

Since the study by Finn et al. (2018), which highlighted the role of the stimulus orienting function on IRAP performance, several research has applied the same rationale in interpreting IRAP results (e.g., Bortoloti et al., 2019; Finn \& Barnes-Holmes, 2019; Kavanagh, Barnes-Holmes, Barnes-Holmes, McEnteggart, \& Finn, 2018; Kavanagh et al., 2019). These interpretations have been based on inferences derived from post-hoc analysis of IRAP performances, without a direct measurement of the orientation function of the stimuli that were presented to the participants. The current study explored the relationship between IRAP effects (e.g., the STTDE) and an independent measure of orienting functions of label and target stimuli presented in the IRAP tasks. Results from the current experiment corroborate the hypothesis that the stimulus orienting function plays an important role on IRAP performance and as a result they are supportive to the DAARRE model.

Acknowledgements This work was part of the research program of Instituto Nacional de Ciência e Tecnologia sobre Comportamento, Cognição e Ensino (National Institute of Science and Technology on Behavior, Cognition, and Teaching), under the leadership of Deisy G. de Souza and supported by the São Paulo Research Foundation (FAPESP, Grant \# 2014/50909-8) and Conselho Nacional de Desenvolvimento Científico e Tecnológico (CNPq, Grant \# 465686/ 2014-1). 
Compliance with Ethical Standards This manuscript has not been published or presented elsewhere, in part or in its entirety, and is not under consideration by another journal.

Availability of Data and Materials Data supporting the findings presented in this article can be found at https://osf.io/qnvbh/ and through doi:10. 17605/OSF.IO/QNVBH.

Conflict of Interest Statement The authors declare that there is no conflict of interest regarding the publication of this article.

Ethical Approval All procedures performed involving human participants were in accordance with the ethical standards of the institutional and national research committee, as well as with the 1964 Helsinki declaration and its later amendments or comparable ethical standards.

Informed Consent Proper informed consent was obtained, and the study design was approved by the appropriate ethics review boards. All the authors have approved the manuscript and agree with submission to the journal.

\section{References}

Barnes-Holmes, D., Barnes-Holmes, Y., Power, P., Hayden, E., Milne, R., \& Stewart, I. (2006). Do you know what you really believe? Developing the Implicit Relational Evaluation Procedure (IRAP) as a direct measure of implicit beliefs. The Irish Psychologist, 32, 169-177.

Barnes-Holmes, D., Barnes-Holmes, Y., Stewart, I., \& Boles, S. (2010a). A sketch of the Implicit Relational Assessment Procedure (IRAP) and the Relational Elaboration and Coherence (REC) model. The Psychological Record, 60, 527-542.

Barnes-Holmes, D., Murtagh, L., Barnes-Holmes, Y., \& Stewart, I. (2010b). Using the Implicit Association Test and the Implicit Relational Assessment Procedure to measure attitudes toward meat and vegetables in vegetarians and meat-eaters. The Psychological Record, 60, 287-305.

Barnes-Holmes, D., Barnes-Holmes, Y., Hussey, I., \& Luciano, C. (2016). Relational Frame Theory: Finding its historical roots and future development. In R. Zettle, S. C. Hayes, D. Barnes-Holmes, \& T. Biglan (Eds.), Wiley handbook of contextual behavioral science (pp. 161-175). Cambridge, UK: Wiley-Blackwell. https://doi.org/ 10.1002/9781118489857.ch8.

Barnes-Holmes, D., Finn, M., McEnteggart, C., \& Barnes-Holmes, Y. (2018). Derived stimulus relations and their role in a behavioranalytic account of human language and cognition. Perspectives on Behavior Science, 41, 155-173. https://doi.org/10.1007/ s40614-017-0124-7.

Bortoloti, R., de Almeida, R. V., de Almeida, J. H., \& de Rose, J. C. (2019). Emotional faces in symbolic relations: A happiness superiority effect involving the equivalence paradigm. Frontiers in Psychology, 10, 1-12. https://doi.org/10.3389/fpsyg.2019.00954.

Bradley, M., \& Lang, P. J. (1994). Measuring Emotion: The SelfAssessment Semantic Differential Manikin and the Semantic Differential Journal of Behavior Therapy \& Experimental Psychiatry, 25, 49-59. https://doi.org/10.1016/0005-7916(94) 90063-9.

Ekman, P., \& Friesen, W. V.(1976). Pictures of Facial Affect. Palo Alto, CA: Consulting Psychologists Press.
Finn, M., Barnes-Holmes, D., \& McEnteggart, C. (2018). Exploring the single-trial-type-dominance-effect in the IRAP: Developing a Differential Arbitrarily Applicable Relational Responding Effects (DAARRE) model. The Psychological Record, 68, 11-25. https:// doi.org/10.1007/s40732-017-0262-z.

Finn, M., Barnes-Holmes, D., \& McEnteggart, C. (2019). Predicting and Influencing the Single Trial-Type Dominance Effect. The Psychological Record, 69, 425-435.

Frischen, A., Eastwood, J. D., \& Smilek, D. (2008). Visual search for faces with emotional expressions. Psychological Bulletin, 134, 662 676. https://doi.org/10.1037/0033-2909.134.5.662.

Gidlöf, K., Anikin, A., Lingonblad, M., \& Wallin, A. (2017). Looking is buying: How visual attention and choice are affected by consumer preferences and properties of the supermarket shelf. Appetite, 116, 29-38. https://doi.org/10.1016/j.appet.2017.04.020.

Greenwald, A. G., McGhee, D. E., \& Schwartz, J. L. K. (1998). Measuring individual differences in implicit cognition: The Implicit Association Test. Journal of Personality \& Social Psychology, 74, 1464-1480. https://doi.org/10.1037/0022-3514.74. 6.1464.

Huziwara, E. M., de Souza, D. d. G., \& Tomanari, G. Y. (2016). Patterns of eye movement in matching-to-sample tasks. Psicologia: Reflexao e Critica, 29, 1-10. https://doi.org/10.1186/s41155-016-0010-3.

Kavanagh, D., Barnes-Holmes, Y., Barnes-Holmes, D., McEnteggart, C., \& Finn, M. (2018). Exploring differential trial type effects and the impact of a read-aloud procedure on deictic relational responding on the IRAP. The Psychological Record, 68, 163-176. https://doi.org/ 10.1007/s40732-018-0276-1.

Kavanagh, D., Matthyssen, N., Barnes-Holmes, Y., Barnes-Holmes, D., McEnteggart, C., \& Vastano, R. (2019). Exploring the use of pictures of self and other in the IRAP: Reflecting upon the emergence of differential trial type effects. International Journal of Psychology \& Psychological Therapy, 19, 323-336.

Krummenacher, J. (2006). Visual search and selective attention. Visual Cognition, 14, 37-41. https://doi.org/10.1080/ 13506280500527676.

Maison, D., Greenwald, A. G., \& Bruin, R. (2001). The Implicit Association Test as a measure of implicit consumer attitudes. Polish Psychological Bulletin, 32, 1-9. https://doi.org/10.1066/ S10012010002.

Maison, D., Greenwald, A. G., \& Bruin, R. H. (2004). Predictive validity of the Implicit Association Test in studies of brands, consumer attitudes, and behavior. Journal of Consumer Psychology, 14, 405-415. https://doi.org/10.1207/s15327663jcp1404_9.

Milosavljevic, M., Navalpakkam, V., Koch, C., \& Rangel, A. (2012). Relative visual saliency differences induce sizable bias in consumer choice. Journal of Consumer Psychology, 22, 67-74. https://doi.org/ 10.1016/j.jcps.2011.10.002.

O'Toole, C., \& Barnes-Holmes, D. (2009). Three chronometric indices of relational responding as predictors of performance on a brief intelligence test: The importance of relational flexibility. The Psychological Record, 59, 119-132.

Treisman, A. M., \& Gelade, G. (1980). A feature-integration theory of attention. Cognitive Psychology, 12, 97-136. https://doi.org/10. 1016/0010-0285(80)90005-5.

Yiend, J. (2010). The effects of emotion on attention: A review of attentional processing of emotional information. Cognition \& Emotion, 24, 3-47. https://doi.org/10.1080/02699930903205698.

Publisher's Note Springer Nature remains neutral with regard to jurisdictional claims in published maps and institutional affiliations. 